\title{
Draft genome sequence of Micromonospora sp. DSW705 and distribution of biosynthetic gene clusters for depsipeptides bearing 4-amino-2,4- pentadienoate in actinomycetes
}

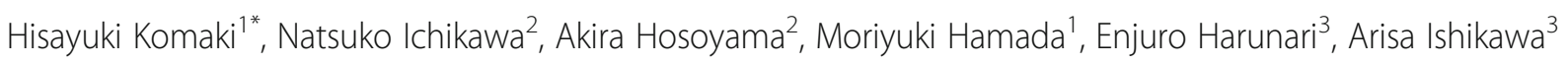 \\ and Yasuhiro Igarashi ${ }^{3}$
}

\begin{abstract}
Here, we report the draft genome sequence of Micromonospora sp. DSW705 (=NBRC 110037), a producer of antitumor cyclic depsipeptides rakicidins $A$ and $B$, together with the features of this strain and generation, annotation, and analysis of the genome sequence. The $6.8 \mathrm{Mb}$ genome of Micromonospora sp. DSW705 encodes 6,219 putative ORFs, of which 4,846 are assigned with COG categories. The genome harbors at least three type I polyketide synthase (PKS) gene clusters, one nonribosomal peptide synthetase (NRPS) gene clusters, and three hybrid PKS/NRPS gene clusters. A hybrid PKS/NRPS gene cluster encoded in scaffold 2 is responsible for rakicidin synthesis. DNA database search indicated that the biosynthetic gene clusters for depsipeptides bearing 4-amino-2,4-pentadienoate are widely present in taxonomically diverse actinomycetes.
\end{abstract}

Keywords: Actinomycete, BE-43547, Micromonospora, Nonribosomal peptide synthetase, Polyketide synthase, Rakicidin, Taxonomy, Vinylamycin

\section{Introduction}

In our screening of antitumor compounds from rare actinomycetes, Micromonospora sp. DSW705 collected from deep seawater was found to produce rakicidins $\mathrm{A}$ and B. Rakicidins are fifteen-membered cyclic depsipeptides comprising three amino acids and a modified fatty acid. The most intriguing feature of rakicidins is the presence of a rare unusual amino acid, 4-amino-2,4-pentadienoate (APDA) in their cyclic structures, which is present only in a limited range of secondary metabolites of actinomycetes [1-3]. To date, five rakicidin congeners have been reported; rakicidins $\mathrm{A}, \mathrm{B}$, and $\mathrm{E}$ were isolated from Micromonospora, and rakicidins $\mathrm{C}$ and $\mathrm{D}$ from Streptomyces [4-7]. Recently, we disclosed the biosynthetic gene (rak) cluster for rakicidin D through the genome analysis of Streptomyces sp. MWW064 and proposed its biosynthetic pathway [8]. In this study, the whole genome shotgun sequencing of Micromonospora sp. DSW705 was conducted to assess its potential in secondary metabolism, to identify the biosynthetic genes for rakicidins $\mathrm{A}$ and $\mathrm{B}$, and to make a comparative analysis with the gene cluster of rakicidin D in Streptomyces sp. MWW064. We here report the draft genome sequence of Micromonospora sp. DSW705, together with the taxonomical identification of the strain, description of its genome properties, and annotation of the rakicidin gene cluster. Furthermore, we investigated distribution of the rak-like clusters in other bacterial strains to evaluate the gene distribution in taxonomically diverse actinomycetes.

\footnotetext{
* Correspondence: komaki-hisayuki@nite.go.jp

${ }^{1}$ Biological Resource Center, National Institute of Technology and Evaluation,

Chiba, Japan

Full list of author information is available at the end of the article
} 


\section{Organism information}

\section{Classification and features}

In the screening of antitumor compounds from rare actinomycetes, Micromonospora sp. DSW705 was isolated from deep seawater collected in Toyama Bay, Japan and found to produce BU-4664 L and rakicidins A and B (unpublished). The general feature of this strain is shown in Table 1. This strain grew well on ISP 2 and ISP 4 agars.

Table 1 Classification and general features of Micromonospora sp. DSW705 [16]

\begin{tabular}{|c|c|c|c|}
\hline MIGS ID & Property & Term & Evidence code $^{a}$ \\
\hline & Classification & Domain Bacteria & TAS [23] \\
\hline & & Phylum Actinobacteria & TAS [24] \\
\hline & & Class Actinobacteria & TAS [25] \\
\hline & & Order Actinomycetales & TAS [25-28] \\
\hline & & $\begin{array}{l}\text { Suborder } \\
\text { Micromonosporineae }\end{array}$ & $\operatorname{TAS}[25,28]$ \\
\hline & & Family Micromonosporaceae & $\operatorname{TAS}[25,27-30]$ \\
\hline & & Genus Micromonospora & $\operatorname{TAS}[27,31]$ \\
\hline & & Species undetermined & - \\
\hline & & Strain DSW705 & IDA \\
\hline & Gram stain & Not tested, likely positive & NAS \\
\hline & Cell shape & Branched mycelia & IDA \\
\hline & Motility & Not reported & \\
\hline & Sporulation & Sporulating & IDA \\
\hline & $\begin{array}{l}\text { Temperature } \\
\text { range }\end{array}$ & Grows from $20^{\circ} \mathrm{C}$ to $45^{\circ} \mathrm{C}$ & IDA \\
\hline & $\begin{array}{l}\text { Optimum } \\
\text { temperature }\end{array}$ & $37^{\circ} \mathrm{C}$ & IDA \\
\hline & $\begin{array}{l}\text { pH range; } \\
\text { Optimum }\end{array}$ & 5 to $8 ; 7$ & IDA \\
\hline & $\begin{array}{l}\text { Carbon } \\
\text { source }\end{array}$ & $\begin{array}{l}\text { Arabinose, fructose, glucose, } \\
\text { raffinose, sucrose, xylose }\end{array}$ & IDA \\
\hline MIGS-6 & Habitat & Sea water & NAS \\
\hline MIGS-6.3 & Salinity & Grows from $0 \%$ to $3 \% \mathrm{NaCl}$ & IDA \\
\hline MIGS-22 & $\begin{array}{l}\text { Oxygen } \\
\text { requirement }\end{array}$ & Aerobic & IDA \\
\hline MIGS-15 & $\begin{array}{l}\text { Biotic } \\
\text { relationship }\end{array}$ & Free-living & IDA \\
\hline MIGS-14 & Pathogenicity & Not reported & \\
\hline MIGS-4 & $\begin{array}{l}\text { Geographic } \\
\text { location }\end{array}$ & Toyama Bay, Japan & NAS \\
\hline MIGS-5 & $\begin{array}{l}\text { Sample } \\
\text { collection }\end{array}$ & October 10, 2005 & NAS \\
\hline MIGS-4.1 & Latitude & Not reported & \\
\hline MIGS-4.2 & Longitude & Not reported & \\
\hline MIGS-4.4 & Altitude & Not reported & \\
\hline
\end{tabular}

${ }^{a}$ Evidence codes - IDA Inferred from Direct Assay, TAS Traceable Author Statement (i.e., a direct report exists in the literature), NAS Non-traceable Author Statement (i.e., not directly observed for the living, isolated sample, but based on a generally accepted property for the species, or anecdotal evidence). These evidence codes are from the Gene Ontology project [32]
On ISP 7 agars, the growth was poor. No growth was observed on ISP 5 agar. No aerial mycelia were observed. Substrate mycelium was orange, turning dark brown on sporulation on ISP 2 agar. No diffusible pigment was observed on ISP 2, ISP 3, ISP 4, ISP 5, ISP 6, and ISP 7 agar media. The strain bored single spore on short sporophore. The spores were spherical (0.7-0.8 $\mu \mathrm{m}$ in diameter) with wrinkle surface. A scanning electron micrograph of the strain is shown in Fig. 1. Growth occurred at 20-45 ${ }^{\circ} \mathrm{C}$ (optimum $37{ }^{\circ} \mathrm{C}$ ) and $\mathrm{pH}$ 5-8 (optimum $\mathrm{pH} 7$ ). Strain DSW705 exhibited growth with $0-3 \%(w / v) ~ N a C l$ (optimum $0 \% \mathrm{NaCl})$. Strain DSW705 utilized arabinose, fructose, glucose, raffinose, sucrose, and xylose for growth. This strain was deposited in the NBRC culture collection with the registration number of NBRC 110037. The genes encoding 16S rRNA were amplified by PCR using two universal primers, $9 \mathrm{~F}$ and 1541R. After purification of the PCR product by AMPure (Beckman Coulter), the sequencing was carried out according to an established method [9]. Homology search of the sequence by EzTaxon-e [10] indicated the highest similarity (99.66\%, 1448/1453) to Micromonospora chalcea DSM $43026^{\mathrm{T}}$ (X92594) as the closest type strain. A phylogenetic tree was reconstructed using ClustalX2 [11] and NJPlot [12] on the basis of the 16S rRNA gene sequence together with those of taxonomically close type strains showing over $98.5 \%$ similarities. Evolutionary distances were calculated using Kimura's two-parameter model [13]. The tree has been deposited into TreeBase (http:// purl.org/phylo/treebase/phylows/study/TB2:S19405). In the phylogenetic tree, strain DSW705 and $M$. chalcea DSM $43026^{\mathrm{T}}$ (X92594) formed a monophyletic cluster with a bootstrap resampling value of $100 \%$ (Fig. 2).

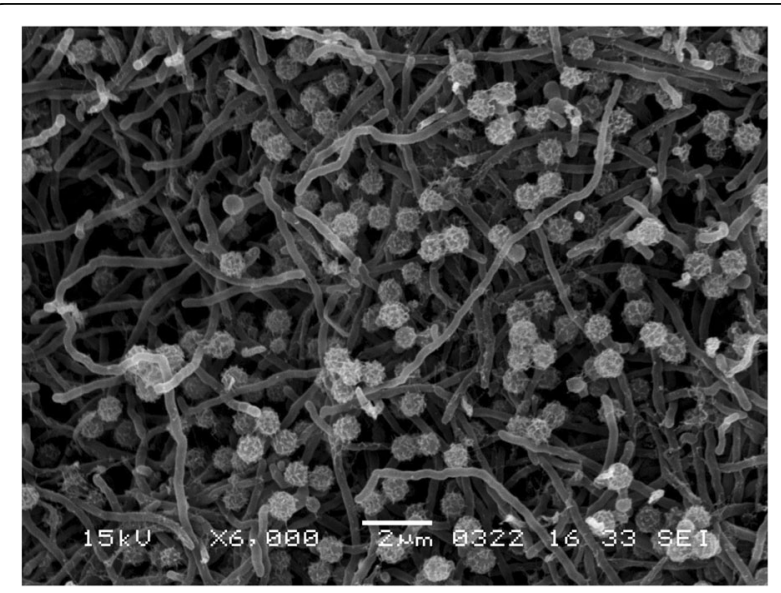

Fig. 1 Scanning electron micrograph of Micromonospora sp. DSW705 grown on $1 / 2$ ISP 2 agar for 7 days at $28^{\circ} \mathrm{C}$. Bar, $2 \mu \mathrm{m}$ 


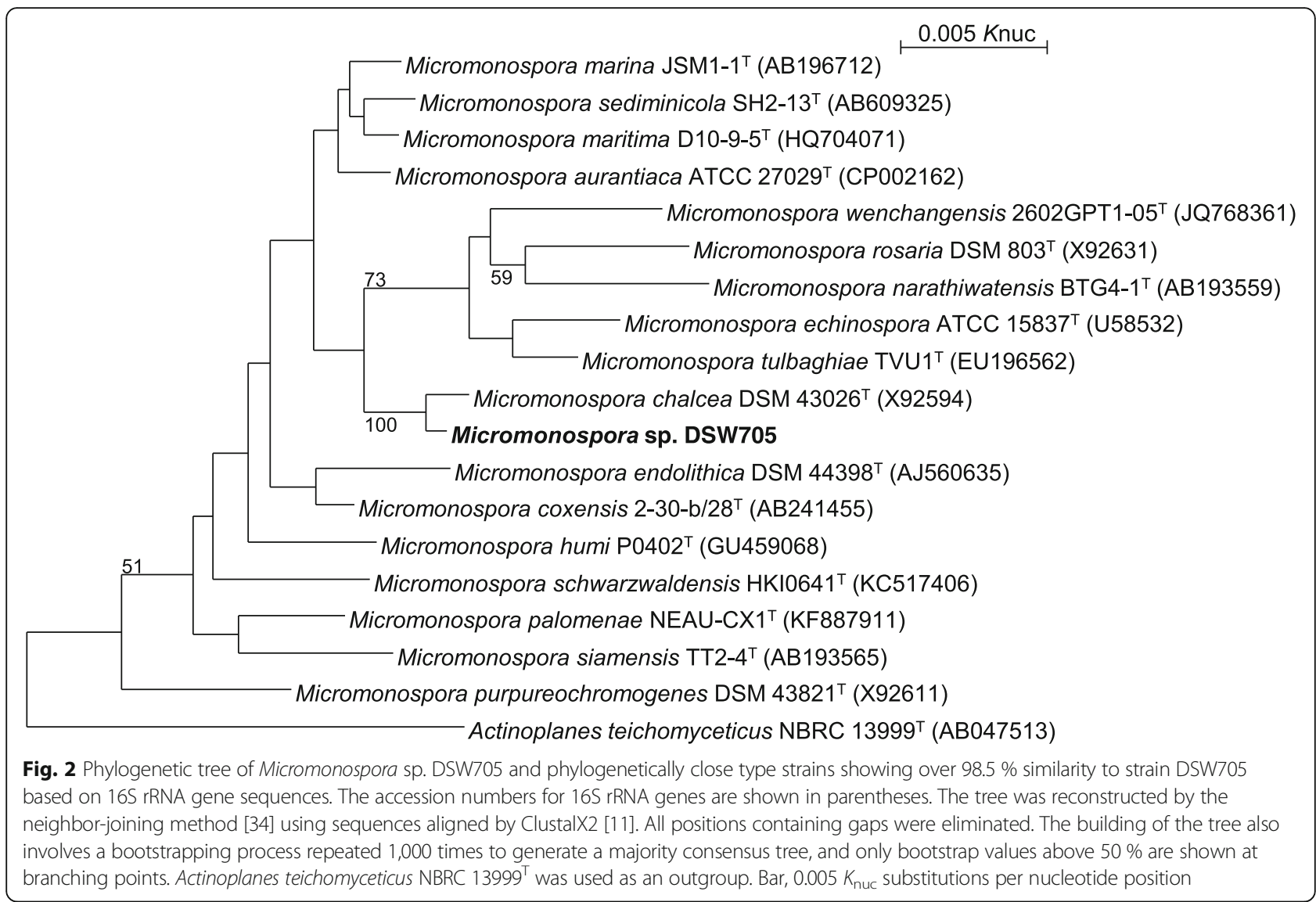

\section{Chemotaxonomic data}

The isomer of diaminopimelic acid in the whole-cell hydrolysate was analyzed according to the method described by Hasegawa et al. [14]. Isoprenoid quinones and cellular fatty acids were analyzed as described previously [15]. The whole-cell hydrolysate of strain DSW705 contained meso-diaminopimelic acid as its diagnostic peptidoglycan diamino acid. The predominant menaquinone was identified as MK-10( $\left.\mathrm{H}_{4}\right)$; MK-9 $\left(\mathrm{H}_{4}\right)$, MK- $10\left(\mathrm{H}_{2}\right)$, and MK$10\left(\mathrm{H}_{6}\right)$ were also detected as minor components. The major cellular fatty acids were found to be iso- $\mathrm{C}_{16: 0}$, iso$\mathrm{C}_{15: 0}$ and anteiso- $\mathrm{C}_{17: 0}$.

\section{Genome sequencing information \\ Genome project history}

In collaboration between Toyama Prefectural University and NBRC, the organism was selected for genome sequencing to elucidate the rakicidin biosynthetic pathway. The draft genome sequences have been deposited in the INSDC database under the accession number BBVA01000001-BBVA01000024. The project information and its association with MIGS version 2.0 compliance are summarized in Table 2 [16].
Growth conditions and genomic DNA preparation

Micromonospora sp. DSW705 was deposited in the NBRC culture collection with the registration number of NBRC 110037. The monoisolate of strain DSW705 was grown on a polycarbonate membrane filter (Advantec) on double

Table 2 Project information

\begin{tabular}{lll}
\hline MIGS ID & Property & Term \\
\hline MIGS 31 & Finishing quality & Improved-high-quality draft \\
MIGS-28 & Libraries used & $\begin{array}{l}\text { 454 shotgun library, Illumina } \\
\text { paired-end library }\end{array}$ \\
MIGS 29 & Sequencing platforms & 454 GS FLX+, Illumina HiSeq1000 \\
MIGS 31.2 & Fold coverage & $5 \times, 100 \times$, respectively \\
MIGS 30 & Assemblers & Newbler v2.6, GenoFinisher \\
MIGS 32 & Gene calling method & Progidal \\
& Locus tag & MSP03 \\
& GenBank ID & BBVA00000000 \\
& GenBank date of release & March 30, 2016 \\
& GOLD ID & Not registered \\
& BioProject & PRJDB3540 \\
MIGS 13 & Source material identifier & NBRC 110037 \\
& Project relevance & Industrial \\
\hline
\end{tabular}


Table 3 Genome statistics

\begin{tabular}{lll}
\hline Attribute & Value & \% of Total \\
\hline Genome size (bp) & $6,795,311$ & 100.0 \\
DNA coding (bp) & $6,219,133$ & 91.5 \\
DNA G + C (bp) & $4,955,456$ & 72.9 \\
DNA scaffolds & 24 & - \\
Total genes & 6,273 & 100.0 \\
Protein coding genes & 6,219 & 99.1 \\
RNA genes & 54 & 0.9 \\
Pseudogenes & - & - \\
Genes in internal clusters & 2,376 & 37.8 \\
Genes with function prediction & 3,909 & 62.3 \\
Genes assigned to COGs & 4,846 & 77.2 \\
Genes with Pfam domains & 5,528 & 84.1 \\
Genes with signal peptides & 480 & 7.7 \\
Genes with transmembrane helices & 1,546 & 24.6 \\
CRISPR repeats & 0 & -
\end{tabular}

Table 4 Number of genes associated with general COG functional

\begin{tabular}{llll}
\multicolumn{3}{c}{ categories } \\
\hline Code & Value & $\%$ age & Description \\
\hline A & 234 & 4.8 & Translation, ribosomal structure and biogenesis \\
K & 1 & 0.02 & RNA processing and modification \\
L & 285 & 12.5 & Transcription \\
B & 2 & 0.9 & Replication, recombination and repair \\
D & 63 & 1.3 & Chromatin structure and dynamics \\
& & & partitioning \\
V & 125 & 2.6 & Defense mechanisms \\
T & 315 & 6.5 & Signal transduction mechanisms \\
M & 281 & 5.8 & Cell wall/membrane biogenesis \\
N & 37 & 0.76 & Cell motility \\
U & 77 & 1.6 & Intracellular trafficking and secretion \\
O & 174 & 3.6 & Posttranslational modification, protein \\
& & & turnover, chaperones \\
C & 345 & 7.1 & Energy production and conversion \\
G & 475 & 9.8 & Carbohydrate transport and metabolism \\
E & 587 & 12.1 & Amino acid transport and metabolism \\
F & 110 & 2.2 & Nucleotide transport and metabolism \\
H & 221 & 4.5 & Coenzyme transport and metabolism \\
I & 277 & 5.7 & Lipid transport and metabolism \\
P & 344 & 7.1 & Inorganic ion transport and metabolism \\
Q & 282 & 5.8 & Secondary metabolites biosynthesis, \\
& & & transport and catabolism \\
R & 984 & 20.3 & General function prediction only \\
S & 457 & 9.4 & Function unknown \\
- & 1,373 & 28.3 & Not in COGs \\
\hline The & & & \\
& 285 &
\end{tabular}

The total is based on the total number of protein coding genes in the genome diluted NBRC 227 agar medium $(0.2 \%$ yeast extract, $0.5 \%$ malt extract, $0.2 \%$ glucose, $2 \%$ agar, pH 7.3) at $28{ }^{\circ} \mathrm{C}$. High quality genomic DNA for sequencing was isolated from the mycelia using an EZ1 DNA Tissue Kit and a Bio Robot EZ1 (Qiagen) according to the protocol for extraction of nucleic acid from Gram-positive bacteria. The size, purity, and double-strand DNA concentration of the genomic DNA were measured by pulsed-field gel electrophoresis, ratio of absorbance values at $260 \mathrm{~nm}$ and $280 \mathrm{~nm}$, and Quant-iT PicoGreen dsDNA Assay Kit (Life Technologies), respectively, to assess the quality of genomic DNA.

\section{Genome sequencing and assembly}

Shotgun and paired-end libraries were prepared and subsequently sequenced using 454 pyrosequencing technology and HiSeq1000 (Illumina) paired-end technology, respectively (Table 2). The $36 \mathrm{Mb}$ shotgun sequences and $682 \mathrm{Mb}$ paired-end sequences were assembled using Newbler v2.6 and subsequently finished using GenoFinisher [17] to yield 24 scaffolds larger than $500 \mathrm{bp}$. The N50 was 629,027 bp.

\section{Genome annotation}

Coding sequences were predicted by Prodigal [18] and tRNA-scanSE [19]. The gene functions were annotated by an in-house genome annotation pipeline, and searched for domains related to polyketide synthase (PKS) and nonribosomal peptide synthetase (NRPS) using the SMART and PFAM domain databases. PKS and NRPS gene clusters and their domain organizations

Table 5 Modular PKS and NRPS gene clusters in Micromonospora sp. DSW705

\begin{tabular}{|c|c|c|c|c|}
\hline $\begin{array}{l}\text { Gene } \\
\text { cluster }\end{array}$ & Encoded in & $\begin{array}{l}\text { No. of } \\
\text { modular } \\
\text { PKS and } \\
\text { NRPS genes }\end{array}$ & $\begin{array}{l}\text { No. of } \\
\text { modules }\end{array}$ & $\begin{array}{l}\text { Backbone of } \\
\text { predicted } \\
\text { product }\end{array}$ \\
\hline pks/nrps-1 (rak) & scaffold 2 & 6 & 7 & $\begin{array}{l}R-C_{3}-C_{3}^{a}-S e r-C_{2}- \\
G l y-X\end{array}$ \\
\hline pks/nrps-2 & scaffold 2 & 6 & 6 & $X-X-X-?-C_{2}-S e r$ \\
\hline pks/nrps-3 & scaffold 2 & 5 & 6 & $X-X-?-C_{2}-A s n-S e r$ \\
\hline pks-1 & scaffold 2 & 12 & 33 & $\begin{array}{l}\mathrm{R}-\mathrm{C}_{2}-\mathrm{C}_{3}-C_{2}-C_{2}-C_{2^{-}} \\
\mathrm{C}_{2}-C_{2}-C_{4}-C_{2}-C_{2}-C_{2} \\
C_{2}-C_{2}-C_{2}-C_{2}-C_{2}-C_{2}- \\
C_{2}-C_{2}-C_{3}-C_{2}-C_{2}-C_{3}- \\
C_{2}-C_{3}-C_{3}-C_{2}-C_{3}-C_{3} \\
C_{3}-C_{2}-C_{2}\end{array}$ \\
\hline pks-2 & scaffold 5 & 1 & 1 & $C_{2}$ \\
\hline pks-3 & scaffold 24 & 1 & 1 & $C_{2}$ \\
\hline nrps-1 & scaffold 2 & 2 & 2 & X-Ala \\
\hline
\end{tabular}

$R$ starter molecule, $C_{3} C_{3}$ unit derived from methylmalonyl-CoA, $C_{2} C_{2}$ unit derived from malonyl-CoA, $X$ amino acid unpredicted, ? lack of $A$ domain in the NRPS module, $C_{4} C_{4}$ unit derived from ethylmalonyl-CoA or methoxymaronylCoA, $C$ ? substrate of AT domain was not predicted

${ }^{a}$ Although antiSMASH predicted that the AT domain incorporates malonyl-CoA as the substrate, the signature sequence for substrate determination is not HAFHS for malonyl-CoA but TSSHS likely for methylmaronyl-CoA [33] 


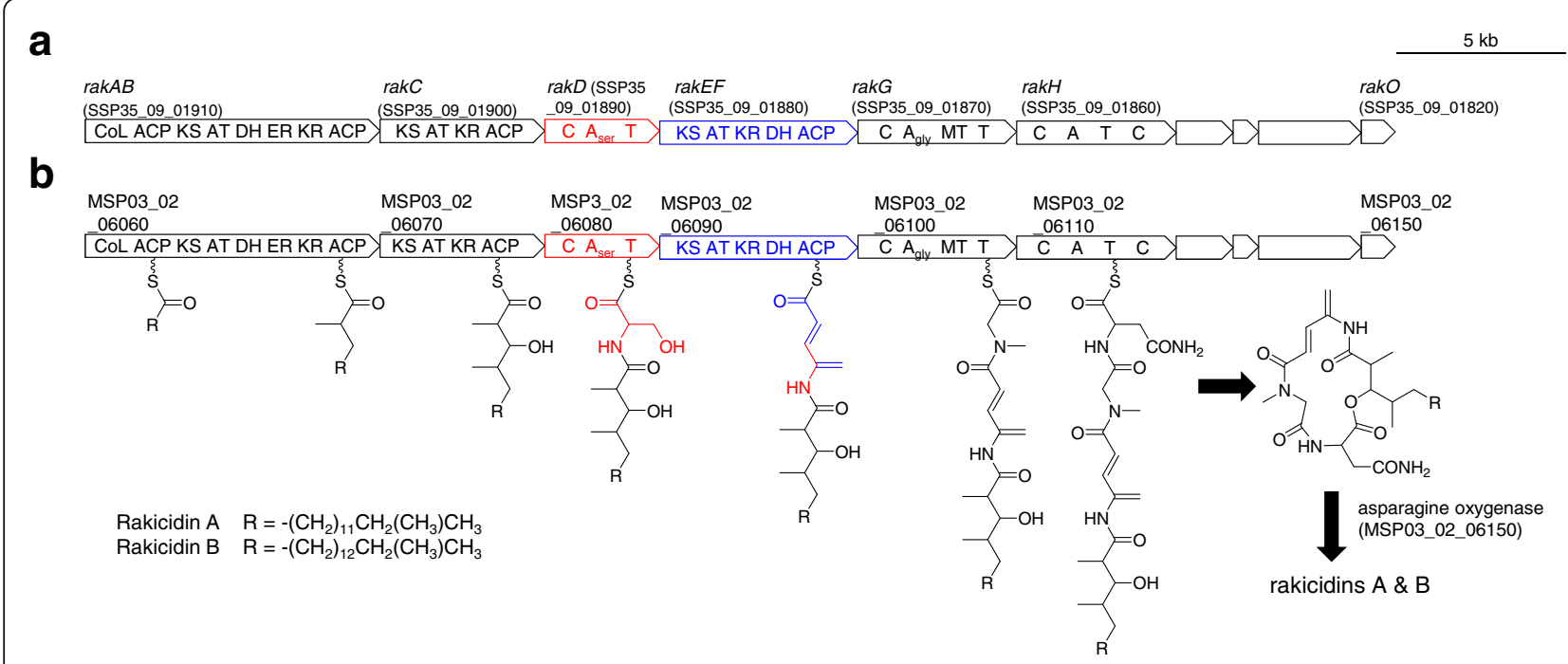

Fig. 3 Genetic map of rakicidin biosynthetic gene cluster of Streptomyces sp. MWW064 (a) and Micromonospora sp. DSW705 and the biosynthetic mechanism of rakicidins $A$ and $B(\mathbf{b})$

were determined as reported previously [9] and using antiSMASH [20]. Substrates of adenylation (A) and acyltransferase (AT) domains were predicted using antiSMASH. BLASTP search against the NCBI $\mathrm{nr}$ databases were also used for predicting function of proteins encoded in the rak cluster.

\section{Genome properties}

The total size of the genome is $6,795,311 \mathrm{bp}$ and the GC content is $72.9 \%$ (Table 3), similar to other genome- sequenced Micronomospora members. Of the total 6,273 genes, 6,219 are protein-coding genes and 54 are RNA genes. The classification of genes into COGs functional categories is shown in Table 4. As for secondary metabolite pathways by modular PKSs and NRPSs, Micromonospora sp. DSW705 has at least three hybrid PKS/NRPS gene clusters, three type I PKS gene clusters, and one NRPS gene clusters. According to the assembly line mechanism [21], we predicted the chemical structures which each cluster would synthesize (Table 5), suggesting the

Table 6 ORFs in the rakicidin-biosynthetic gene cluster of Micromonospora sp. DSW705

\begin{tabular}{llllll}
\hline $\begin{array}{l}\text { MSP03_02_ } \\
\text { (locus tag) }\end{array}$ & Size (aa) & Deduced function & Protein homolog [origin] & $\begin{array}{l}\text { Identity/ } \\
\text { similarity (\%) }\end{array}$ & $\begin{array}{l}\text { Accession } \\
\text { number }\end{array}$ \\
\hline 06020 & 1,046 & $\begin{array}{l}\text { Transcriptional } \\
\text { regulator }\end{array}$ & Transcriptional regulator [Micromonospora purpureochromogenes] & $95 / 95$ & WP_030498969 \\
06030 & 564 & Monooxygenase & Monooxygenase [Micromonospora purpureochromogenes] & $94 / 95$ & WP_030498970 \\
06040 & 314 & Unknown & Hypothetical protein [Salinispora pacifica] & $66 / 75$ & WP_027650590 \\
06050 & 674 & Unknown & LigA protein [Micromonospora sp. M42] & $99 / 99$ & EWM62996 \\
06060 & 2,944 & PKS & Hypothetical protein [Micromonospora purpureochromogenes] & $95 / 96$ & WP_036342114 \\
06070 & 1,608 & PKS & Non-ribosomal peptide synthetase [Micromonospora sp. M42] & $93 / 93$ & EWM63000 \\
06080 & 1,123 & NRPS & Non-ribosomal peptide synthetase [Micromonospora sp. M42] & $99 / 100$ & EWM63002 \\
06090 & 1,883 & PKS & Beta-ketoacyl synthase [Micromonospora purpureochromogenes] & $97 / 97$ & WP_030498975 \\
06100 & 1,517 & NRPS & Hypothetical protein, partial [Micromonospora purpureochromogenes] & $97 / 97$ & WP_036342201 \\
06110 & 1,563 & NRPS & Hypothetical protein [Micromonospora purpureochromogenes] & $95 / 95$ & WP_030498977 \\
06120 & 570 & ABC transporter & Pyoverdine ABC transporter permease/ATP-binding protein & $100 / 100$ & EWM63008 \\
06130 & 287 & Type-II thioesterase & Gramicidin S biosynthesis protein GrsT [Micromonospora sp. M42] & $98 / 98$ & EWM63009 \\
06140 & 955 & NRPS & Non-ribosomal peptide synthetase [Micromonospora sp. M42] & $99 / 99$ & EWM63010 \\
06150 & 329 & Asparagine & Clavaminate synthase [Micromonospora sp. M42] & $100 / 100$ & EWM63011 \\
06160 & 771 & Transporter & Membrane protein mmpL11 [Micromonospora sp. M42] & $99 / 99$ & EWM63012 \\
\hline
\end{tabular}


potential of Micromonospora sp. DSW705 to produce diverse polyketide- and nonribosomal peptide-compounds as secondary metabolites.

\section{Insights from the genome sequence Rakicidin biosynthetic gene cluster in Micromonospora sp. DSW705}

Our previous study revealed that rakicidin is synthesized by a hybrid PKS/NRPS gene cluster. Its domain organization is shown in Fig. 3a [8]. Among the three hybrid PKS/NRPS gene clusters present in the Micromonospora sp. DSW705 genome shown in Table 5, only pks/ nrps-1 shows the same domain organization as the rak cluster of Streptomyces sp. MWW064 (Fig. 3b). Since this gene cluster encodes all the enzymes necessary for assembling the rakicidin core structure, this cluster was confirmed as a rak cluster (Table 6). Gene organizations of the clusters for rakicidin D in Streptomyces sp. MWW064 (Fig. 3a) and rakicidins A and $\mathrm{B}$ in Micromonospora sp. DSW705 (Fig. 3b) are a rakicidins

Micromonospora sp. DSW705

Streptomyces sp. MWW064

M. purpureochromogenes NRRL B-2672

Micromonospora sp. M42

b vinylamycin-related

"Str. rubellomurinus" ATCC 31215

Frankia sp. ACN1ag

Frankia sp. Cpl1-P

Frankia sp. Cpl1-S

Str. davawensis JCM 4913

Str. vitaminophilus DSM $41686^{\top}$

Streptomyces sp. CNH099

Streptomyces sp. CNQ-509

Str. durhamensis NRRL-ISP-5539

Str. griseolus NRRL B-2925

Str. halstedii NRRL ISP-5068'

Streptomyces sp. DpondAA-B6

Streptomyces sp. NRRL S-1521

Streptomyces sp. NTK973

Streptomyces sp. WMMB 714

Streptomyces sp. 769

Streptomyces sp. MspMP-M5

Nocardiposis sp. CNS639

\section{C $\mathrm{BE}-43547$}

Salinispora arenicola CNR107

Micromonospora sp. RV43

\section{d others}

Kitasatospora griseola MF730-N6 Str. purpeofuscus NRRL B-1817 Streptomyces sp. NRRL F-6131

Streptomyces sp. XY431

Kitasatospora sp. MBT66

Str. celluloflavas NRRL B-2493 ${ }^{\top}$

Str. albus subsp. albus NRRL B-2513

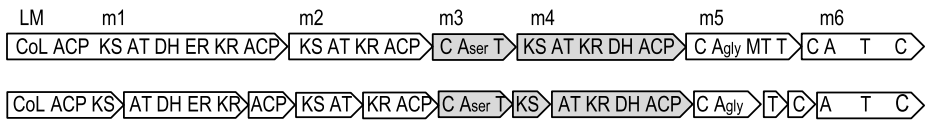

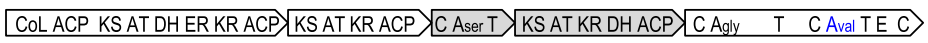

CoL ACP KS AT DH ER KR ACP KS AT KR ACP C Aser T KS AT KR DH ACP C Agly $\quad$ T C Aval TE C

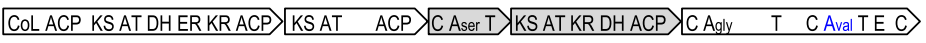

CoL ACP KS AT DHER KR ACP KS AT ACP C Aser T KS AT KR DH ACP...

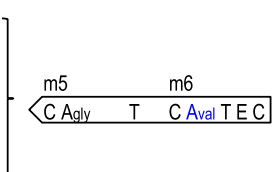

CoL ACP KS AT DH ER KR ACP KS AT ACP C Aser T KS AT KR DH ACP

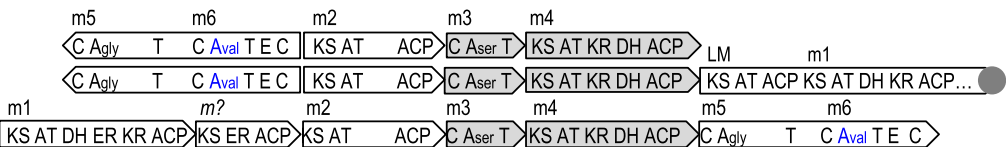

Fig. 4 Hybrid PKS/NRPS gene clusters for depsipeptides bearing 4-amino-2,4-pentadienoate (APDA) moieties in published genome sequences of actinomycete strains. Gene clusters for rakicidins (a), vinylamycin-related compounds (b), BE-43547 (c), and others (d). NRPS and PKS genes for the synthesis of APDAs are shaded in light gray. Terminals of scaffold sequences are shown in dark gray circles. Locus tag numbers of ORFs in this figure are as follows: Micromonospora purpureochromogenes NRRL B-2672, IH31_RS0100575 to IH31_RS0100600; Micromonospora sp. M42, MCBG_00130 to MCBG_00140; "Streptomyces rubellomurinus" ATCC 31215, VM95_RS28100 to VM95_RS28120; Frankia sp. ACN1ag, UK82_23055 to UK82_23085; Frankia sp. Cpl1-P, FF86_101835 to FF86_101841; Frankia sp. Cpl1-S, FF36_02633 to FF36_02639; Streptomyces davawensis JCM 4913, BN159_0686 to BN159_0681; Streptomyces vitaminophilus DSM 41686' , A3IG_RS0122990 to A3IG_RS0122970; Streptomyces sp. CNH099, B121_RS0112700 to B121_RS0112685 and B121_RS37950; Streptomyces sp. CNQ-509, AA958_29290 to AA958_29325; Streptomyces durhamensis NRRL-ISP-5539', IO33_RS0129710 to IO33_RS0129695; Streptomyces griseolus NRRL B-2925 ', IH14_RS0112325 to IH14_RS0112355; Streptomyces halstedii NRRL ISP-5068', IG73_RS0111725 to IG73_RS0111755; Streptomyces sp. DpondAA-B6, K379_RS0125155 to K379_RS0125185; Streptomyces sp. NRRL S-1521, ADL30_05665 to ADL30_05635; Streptomyces sp. NTK973, DT87_RS01535 to DT87_RS01505; Streptomyces sp. WMMB 714, H181_RS01075 to H181_RS01105; Streptomyces sp. 769, GZL_RS00255 to GZL_RS00285; Streptomyces sp. MspMP-M5, B073_RS0123900 to B073_RS40860; Nocardiposis sp. CNS639, G011_RS0119410 to G011_RS0119385; Salinispora arenicola CNR107, F583_RS01000000127215 to F583_RS01000000127205; Micromonospora sp. RV43, ABD52_RS02395 to ABD52_RS02415; Kitasatospora griseola MF730-N6, TR51_RS11025 to TR51_RS11045; Streptomyces purpeofuscus NRRL B-1817', IF01_RS0123020 to IF01_RS0123045; Streptomyces sp. NRRL F-6131, IF39_RS0107420 to IF39_RS0107445; Streptomyces sp. XY431, ADK60_02665 to ADK60_02635; Kitasatospora sp. MBT66, BI06_RS24475 to BI06_RS24440; Streptomyces celluloflavas NRRL B-2493', IH09_RS02990 to IH09_RS03015; Streptomyces albus subsp. albus NRRL B-2513, ACZ90_11100 to ACZ90_11120 
essentially identical. Proposed biosynthetic pathway for rakicidins in Micromonospora sp. DSW705 is illustrated in Fig. 3b.

\section{Biosynthetic gene clusters for rakicidins and the related compounds in other strains}

Since the BLAST analysis shown in Table 6 suggests that other Micromonospora strains such as $M$. purpureochromogenes and Micromonospora sp. M42 may possess rak clusters, hybrid PKS/NRPS gene clusters similar to rak clusters were searched for bacterial strains whose genome sequences and the ORF information are available in the GenBank database. We carried out BLAST search using RakEF sequence of Micromonospora sp. DSW705 and Streptomyces sp. MWW064 as the queries, and then analyzed each of the gene clusters encoding RakEF orthologues using antiSMASH [20] and manually if necessary. As shown in Fig. 4, three Micromonospora, 19 Streptomyces, three Frankia, one Nocardiopisis, one Salinispora, and two Kitasatospora strains were found to possess hybrid PKS/NRPS gene clusters encoding RakEF orthologues. On the basis of the domain organizations and amino-acids substrates of A domains, these gene clusters can be classified into four groups (Fig. 4).

M. purpureochromogenes NRRL B-2672 harbors a rak cluster as same as Micromonospora sp. DSW705 and Streptomyces sp. MWW064. Micromonospora sp. M42 also possesses almost the same cluster, but the methyltransferase (MT) domain in module 5 (m5) is not present and some ORFs are fragmented (Fig. 4a).

Eighteen gene clusters categorized into Fig. $4 \mathrm{~b}$ have domain organizations similar to rak clusters but the substrate of A domain in $\mathrm{m} 6$ was predicted to be $\mathrm{L}$ valine. As vinylamycin and microtermolide contain a valine residue in their depsipeptide structure [1, 2], the four gene clusters of "Streptomyces rubellomurinus" ATCC 31215 and three Frankia strains were proposed to be responsible for vinylamycin biosynthesis. A plausible biosynthetic pathway for vinylamycin is illustrated in Fig. 5a. If the loading modules incorporate a $\mathrm{C}_{3}$ unit or LMs encode an AT domain for a $\mathrm{C}_{3}$ starter instead of the CoA-ligase domain, the

\section{a}

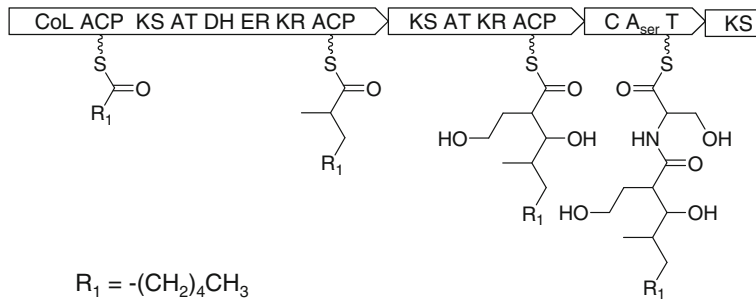

b

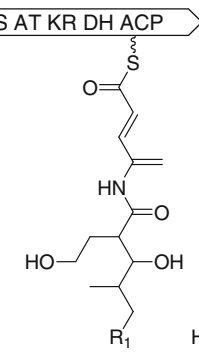

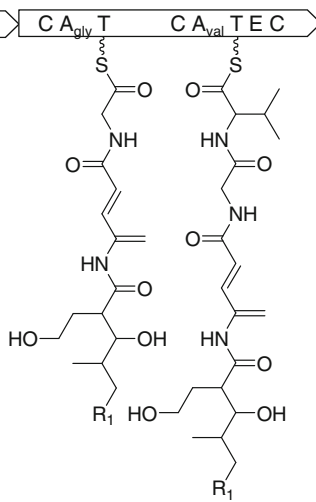

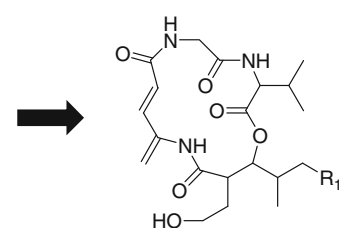

vinylamycin

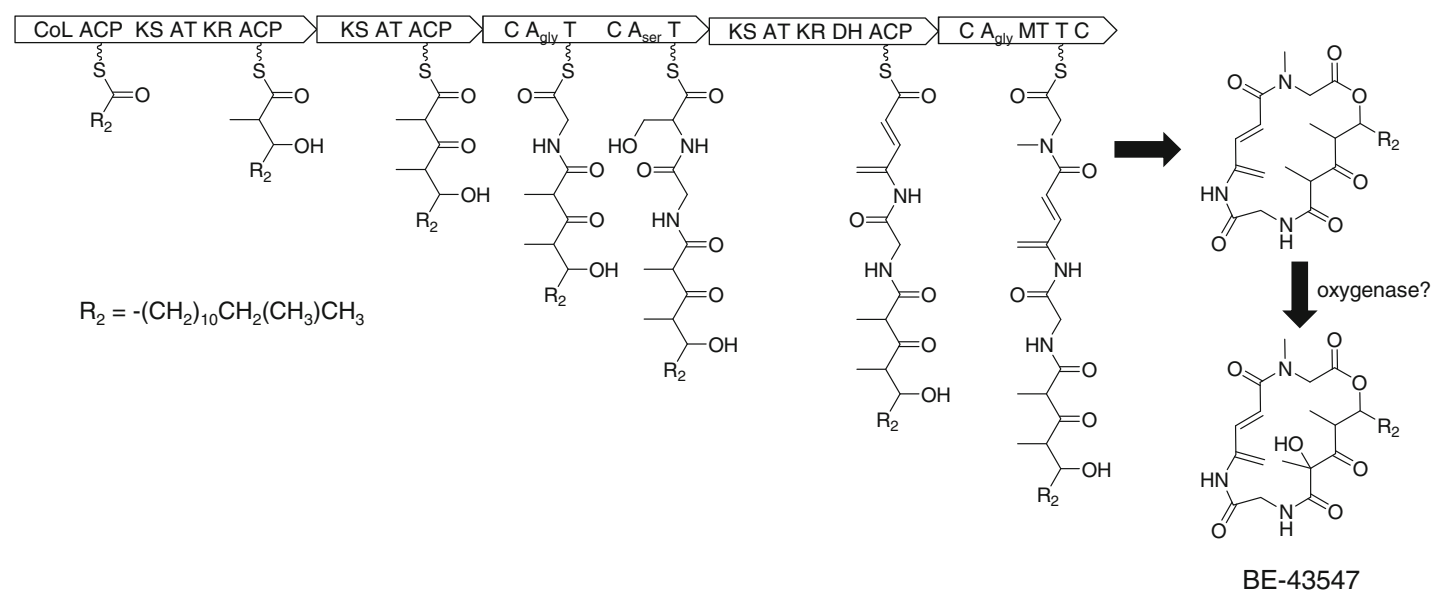

Fig. 5 Putative biosynthetic pathways for vinylamycin (a) and BE-43547 (b) 
cluster is likely responsible for microtermolide biosynthesis. The remaining 14 strains in Fig. $4 \mathrm{~b}$ lack a KR domain in $\mathrm{m} 2$. In the clusters of eight among the 18 strains, NRPSs for $\mathrm{m} 5$ and $\mathrm{m} 6$ are encoded the complementary strands, although the cluster of Streptomyces durhamensis NRRL ISP- $5539^{\mathrm{T}}$ was not completely sequenced. Streptomyces sp. 769 does not have the PKS for LM and $\mathrm{m} 1$. In the cluster of Streptomyces sp. MspMP-M5, the PKS likely for LM and $\mathrm{m} 1$ is encoded downstream of the PKS gene for $\mathrm{m} 4$, although the gene cluster was not completely sequenced. The cluster of Nocardiposis sp. CNS639 likely lacks a LM, and some domains are distinct from those of other strains.
Gene clusters of Salinispora arenicola CNR107 and Micromonospora sp. RV43 contain three NRPS modules at $\mathrm{m} 3, \mathrm{~m} 4$, and $\mathrm{m} 6$, which were predicted to incorporate glycine, serine, and glycine, respectively. Only BE-43547 is known as a depsipeptide containing two glycines and APDA moiety. According to the domain organization, these two clusters are proposed to be involved with BE-43547 production as illustrated in Fig. 5b.

Figure $4 \mathrm{~d}$ shows gene clusters in which the last NRPS module incorporates amino acids different from those of the other three groups described above. Five gene clusters shown in green were predicted to incorporate L-tyrosine into the polyketide/nonribosomal peptide chains by $\mathrm{m} 6$. Since depsipeptides bearing

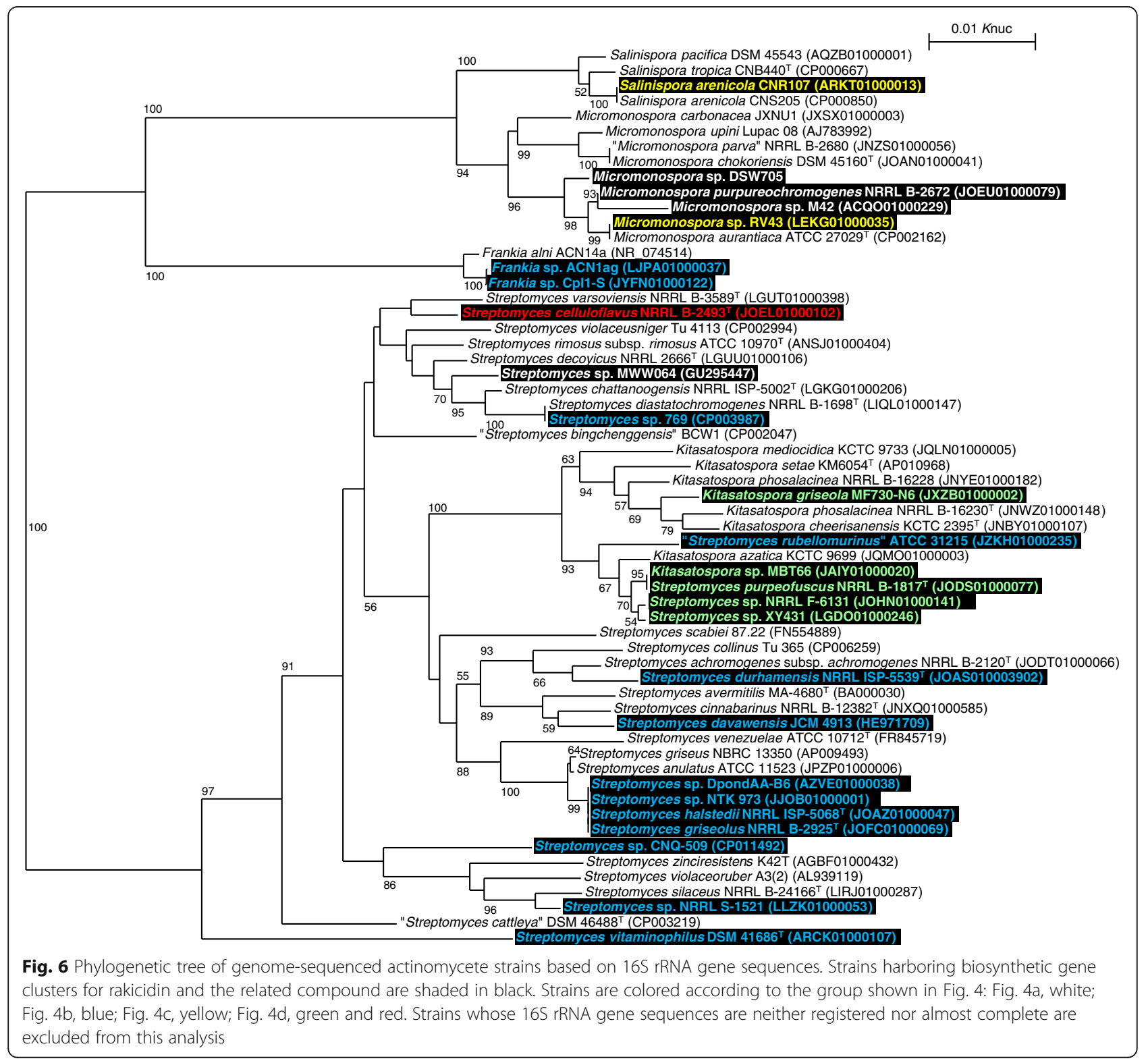


both tyrosine and APDA residues are not known, products from these clusters may be structurally novel. Two gene clusters of Streptomyces celluloflavas NRRL B- $2493^{\mathrm{T}}$ and Streptomyces albus subsp. albus NRRL B-2513 showed the same domain organization as rak clusters, but NRPS substrate prediction suggests incorporation of L-glutamate and L-tryptophan/ $\beta$-hydroxy-tyrosine (bht) by $\mathrm{m} 6$, respectively. Because rakicidin analogues containing these amino acids in place of the asparagine residue have not been reported, production of novel APDA-containing peptides is expected in these strains.

\section{Distribution of the gene clusters among genome-sequenced strains}

Whole genome sequencing has been performed for a large number of actinomycete strains. At present, genome sequences of over 227 Streptomyces species, eight species and six strains of Kitasatospora, eight species and seven strains of Micromonospora, three Salinispora species, one species and 97 strains of Frankia, and 18 species and 6 strains of Nocardiopsis are available from the GenBank database. Among them, 29 strains possess the rak-like gene clusters. To investigate the correlation between evolution and secondary metabolite gene distribution, strains harboring the rak-like gene clusters (shaded in black) were mapped onto the phylogenetic tree of genome-sequenced strains based on 16S rRNA gene sequences (Fig. 6). Micromonospora strains are divided into two clades, one of which includes three rakicidin-producers and one BE-43547-producer. Strain MWW064 is the only Streptomyces that possesses the rak cluster other than Micromonospora. In contrast, vinylamycin-related gene clusters, shown in blue, are distributed in taxonomically diverse Streptomyces strains. It is noteworthy that two Frankia strains have the same gene cluster whereas only four compounds have been described for Frankia species [22]. This genus should be more examined for secondary metabolite production. BE-43547 gene clusters are present only in two strains of two genera belonging to the family Micromonosporaceae in this analysis. But, since this compound was originally found from Streptomyces [3], the gene cluster must also be present in the genus Streptomyces. Presence of gene clusters for depsipeptides containing a tyrosine residue is limited to the genus Kitasatospora and phylogenetically close Streptomyces members. The S. celluloflavas NRRL B-2493 ${ }^{\mathrm{T}}$ gene cluster shows a similar domain organization to those of rak clusters stated above, but this strain is not taxonomically close to rakicidin producers.

\section{Conclusions}

The 6.8 Mb draft genome of Micromonospora sp. DSW705, a producer of rakicidins A and B isolated from deep seawater, has been deposited at GenBank/ENA/
DDBJ under the accession number BBVA00000000. This strain contains seven PKS and NRPS gene clusters, from which rakicidin-biosynthetic gene cluster was identified. Gene clusters for the synthesis of rakicidins or the related compounds are present in taxonomically diverse actinomycete strains, belonging to Micromonospora, Salinispora, Frankia, Nocardiposis, Kitasatospora, and Streptomyces. These findings provide useful information for discovering new and diverse depsipeptides bearing the APDA unit, and accelerate understanding of relationship between taxonomy and secondary metabolite gene distribution, and will possibly provide the insight regarding to the evolution of secondary metabolite genes.

\section{Abbreviations}

A: Adenylation; ABC: ATP-binding cassette; ACP: Acyl carrier protein; APDA: 4amino-2,4-pentadienoate; AT: Acyltransferase; ATP: Adenosine triposphate; bht: $\beta$-hydroxy-tyrosine; C: Condensation; CoA: Coenzyme A; CoL: CoA ligase; DDBJ: DNA Data Bank of Japan; DH: Dehydratase; E: Epimerization;

ER: Enoylreductase; ISP: International Streptomyces project; KR: Ketoreductase; KS: Ketosynthase; LM: Loading module; m: Module; MT: Methyltransferase; NBRC: Biological Resource Center, National Institute of Technology and Evaluation; NRPS: Nonribosomal peptide synthetase; PKS: Polyketide synthase; $\mathrm{T}$ : Thiolation

\section{Acknowledgements}

This research was supported by a Grant-in-aid for Scientific Research from the Ministry of Education, Culture, Sports, and Technology of Japan to Y.I. We also thank Dr. Akane Kimura, Ms. Yuko Kitahashi, Ms. Satomi Saitou and Ms.

Chiyo Shibata for assistance to this study.

\section{Authors' contributions}

HK analysed biosynthetic gene clusters and drafted the manuscript. $\mathrm{NI}$ annotated the genome sequences. AH sequenced the genome. $\mathrm{MH}$ performed chemotaxonomic experiments. EH examined the features of the strain. Al predicted products of gene clusters similar to rak clusters. YI designed this study and edited the manuscript. All authors read and approved the final manuscript.

\section{Competing interests}

The authors declare that they have no competing of interests.

\section{Author details}

${ }^{1}$ Biological Resource Center, National Institute of Technology and Evaluation, Chiba, Japan. ${ }^{2}$ NBRC, Tokyo, Japan. ${ }^{3}$ Biotechnology Research Center and Department of Biotechnology, Toyama Prefectural University, Toyama, Japan.

Received: 1 April 2016 Accepted: 12 October 2016

Published online: 22 October 2016

\section{References}

1. Carr G, Poulsen M, Klassen JL, Hou Y, Wyche TP, Bugni TS, Currie CR, Clardy J. Microtermolides A and B from termite-associated Streptomyces sp. and structural revision of vinylamycin. Org Lett. 2012;14(11):2822-5.

2. Igarashi M, Shida T, Sasaki Y, Kinoshita N, Naganawa H, Hamada M, Takeuchi T. Vinylamycin, a new depsipeptide antibiotic, from Streptomyces sp. J Antibiot. 1999:52(10):873-9.

3. Nishioka H, Nakajima S, Nagashima M, Kojiri K, Suda H. BE-43547 series substances, their manufacture with Streptomyces species, and their use as antitumor agents. Japan Patent. 1998. JP 10147594 A 19980602.

4. Igarashi Y, Shimasaki R, Miyanaga S, Oku N, Onaka H, Sakurai H, Saiki I, Kitani S, Nihira T, Wimonsiravude W, et al. Rakicidin D, an inhibitor of tumor cell invasion from marine-derived Streptomyces sp. J Antibiot. 2010;63(9):563-5.

5. McBrien KD, Berry RL, Lowe SE, Neddermann KM, Bursuker I, Huang S, Klohr SE, Leet JE. Rakicidins, new cytotoxic lipopeptides from Micromonospora sp. fermentation, isolation and characterization. J Antibiot. 1995;48(12):1446-52. 
6. Oku N, Matoba S, Yamazaki YM, Shimasaki R, Miyanaga S, Igarashi Y. Complete stereochemistry and preliminary structure-activity relationship of rakicidin A, a hypoxia-selective cytotoxin from Micromonospora sp. J Nat Prod. 2014;77(11):2561-5.

7. Hu J, Wunderlich D, Sattler I, Feng X, Grabley S, Thiericke R. Rakicidin C, a new cyclic depsipeptide from Streptomyces sp. European J Org Chem. 2000;19:3353-6.

8. Komaki H, Ishikawa A, Ichikawa N, Hosoyama A, Hamada M, Harunari E, Nihira T, Panbangred W, Igarashi Y. Draft genome sequence of Streptomyces sp. MWW064 for elucidating the rakicidin biosynthetic pathway. Stand Genomic Sci. 2016;11(1):83. doi:10.1186/s40793-016-0205-3.

9. Komaki H, Ichikawa N, Hosoyama A, Fujita N, Igarashi Y. Draft genome sequence of marine-derived Streptomyces sp. TP-A0598, a producer of anti-MRSA antibiotic lydicamycins. Stand Genomic Sci. 2015;10:58.

10. Kim OS, Cho YJ, Lee K, Yoon SH, Kim M, Na H, Park SC, Jeon YS, Lee JH, Yi H, et al. Introducing EzTaxon-e: a prokaryotic $16 \mathrm{~S}$ rRNA gene sequence database with phylotypes that represent uncultured species. Int J Syst Evol Microbiol. 2012;62(Pt 3):716-21.

11. Larkin MA, Blackshields G, Brown NP, Chenna R, McGettigan PA, McWilliam H, Valentin F, Wallace IM, Wilm A, Lopez R, et al. Clustal W and Clustal X version 2.0. Bioinformatics. 2007;23(21):2947-8.

12. Perriere G, Gouy M. WWW-query: an on-line retrieval system for biological sequence banks. Biochimie. 1996;78(5):364-9.

13. Kimura M. A simple method for estimating evolutionary rates of base substitutions through comparative studies of nucleotide sequences. J Mol Evol. 1980;16(2):111-20.

14. Hasegawa T, Takizawa M, Tanida S. A rapid analysis for chemical grouping of aerobic actinomycetes. J Gen Appl Microbiol. 1983;29(4):319-22.

15. Hamada M, Yamamura H, Komukai C, Tamura T, Suzuki K, Hayakawa M. Luteimicrobium album sp. nov., a novel actinobacterium isolated from a lichen collected in Japan, and emended description of the genus Luteimicrobium. J Antibiot. 2012;65(8):427-31.

16. Field D, Garrity G, Gray T, Morrison N, Selengut J, Sterk P, Tatusova T, Thomson N, Allen MJ, Angiuoli SV, et al. The minimum information about a genome sequence (MIGS) specification. Nat Biotechnol. 2008;26(5):541-7.

17. Ohtsubo Y, Maruyama F, Mitsui H, Nagata Y, Tsuda M. Complete genome sequence of Acidovorax sp. strain KKS102, a polychlorinated-biphenyl degrader. J Bacteriol. 2012;194(24):6970-1.

18. Hyatt D, Chen GL, Locascio PF, Land ML, Larimer FW, Hauser L. Prodigal: prokaryotic gene recognition and translation initiation site identification. BMC Bioinformatics. 2010;11:119.

19. Lowe TM, Eddy SR. tRNAscan-SE: a program for improved detection of transfer RNA genes in genomic sequence. Nucleic Acids Res. 1997;25(5):955-64.

20. Blin K, Medema MH, Kazempour D, Fischbach MA, Breitling R, Takano E, Weber T. antiSMASH 2.0-a versatile platform for genome mining of secondary metabolite producers. Nucleic Acids Res. 2013;41(Web Server issue):W204-12.

21. Fischbach MA, Walsh CT. Assembly-line enzymology for polyketide and nonribosomal peptide antibiotics: logic, machinery, and mechanisms. Chem Rev. 2006;106(8):3468-96.

22. CRCnetBase. Dictionary of Natural Products on DVD-ROM, version 19:1 London: Chapman \& Hall; 2014.

23. Woese CR, Kandler O, Wheelis ML. Towards a natural system of organisms: proposal for the domains Archaea, Bacteria, and Eucarya. Proc Natl Acad Sci U S A. 1990;87(12):4576-9.

24. Goodfellow M. Phylum XXVI. Actinobacteria phyl. nov. In: Goodfellow M, Kämpfer P, Busse H-J, Trujillo ME, Suzuki K-I, Ludwig W, Whitman WB, editors. Bergey's Manual of Systematic Bacteriology, Second Edition, Volume 5, Part A. New York: Springer; 2012. p. 33.

25. Stackebrandt E, Rainey FA, Ward-Rainey NL. Proposal for a new hierarchic classification system, Actinobacteria classis nov. Int J Syst Bacteriol. 1997:47:479-91.

26. Buchanan RE. Studies in the Nomenclature and Classification of the Bacteria: II. The Primary Subdivisions of the Schizomycetes. J Bacteriol. 1917;2(2):155-64.

27. Skerman VBD, McGowan V, Sneath PHA. Approved lists of bacterial names. Int J Syst Bacteriol. 1980;30:225-420.

28. Zhi XY, Li WJ, Stackebrandt E. An update of the structure and 165 rRNA gene sequence-based definition of higher ranks of the class Actinobacteria with the proposal of two new suborders and four new families and emended descriptions of the existing higher taxa. Int J Syst Evol Microbiol. 2009;59(Pt 3):589-608.
29. Koch C, Kroppenstedt RM, Rainey FA, Stackebrandt E. 16 ribosomal DNA analysis of the genera Micromonospora, Actinoplanes, Catellatospora, Catenuloplanes, Couchioplanes, Dactylosporangium, and Pilimelia and emendation of the family Micromonosporaceae. Int J Syst Bacteriol. 1996:46:765-8.

30. Krasil'nikov NA. Ray fungi and related organisms. Actinomycetales, Akademii Nauk, Moscow. 1938. p. 272

31. Ørskov J. Investigations into the morphology of the ray fungi. Copenhagen: Levin and Munksgaard; 1923.

32. Ashburner M, Ball CA, Blake JA, Botstein D, Butler H, Cherry JM, Davis AP, Dolinski K, Dwight SS, Eppig JT, et al. Gene ontology: tool for the unification of biology. The Gene Ontology Consortium. Nat Genet. 2000;25(1):25-9.

33. Kakavas SJ, Katz L, Stassi D. Identification and characterization of the niddamycin polyketide synthase genes from Streptomyces caelestis. J Bacteriol. 1997;179(23):7515-22.

34. Saitou N, Nei M. The neighbor-joining method: a new method for reconstructing phylogenetic trees. Mol Biol Evol. 1987;4(4):406-25.

\section{Submit your next manuscript to BioMed Central and we will help you at every step:}

- We accept pre-submission inquiries

- Our selector tool helps you to find the most relevant journal

- We provide round the clock customer support

- Convenient online submission

- Thorough peer review

- Inclusion in PubMed and all major indexing services

- Maximum visibility for your research

Submit your manuscript at www.biomedcentral.com/submit
Biomed Central 